\title{
A Review on Trust-Aware and Privacy Preserving Profile Matching System for Personalized User Recommendations in Social networks
}

\author{
Kulkarni Vaishnavi Shripad \\ University of Pune \\ Department of Computer Engg. \\ GES's R. H. Sapat College of Engg
}

\author{
Prof. Archana S. Vaidya \\ University of Pune \\ Department of Computer Engg. \\ GES's R. H. Sapat College of Engg
}

\begin{abstract}
Trust is becoming a very important part of social network from the security point of view. In this project, we introduce a framework for handling trust in social networks, which is based on reputation mechanism. The reputation mechanism captures the implicit and explicit connections between the network members, analyses the semantics and dynamics of these connections, and provides personalized user recommendations to the network members. Based on the trust semantics, the system will provide the positive (trustworthy) recommendations and the negative (untrustworthy) recommendations. Along with this our system provides one more interesting mode i.e. public profile matching that preserves privacy on social networks. This profile matching contributes in reputation ratings required for suggestions of friend list. In order to compute the reputation of each member, we adopt several other properties of trust such as, transitivity, personalization, and context, and draw ideas from sociology axioms. Trust is not perfectly transitive in social networks, in that trust decays along the transition path, but it is generally agreed that it can be communicated between people. Along with trust generation percentile of profile matching is also considered for personal recommendation.
\end{abstract}

\section{Keywords}

Social Networks, Reputation, Personalisation, Trust, Recommendation, Profile Matching.

\section{INTRODUCTION}

Social network analysis has been a major area of research for sociologists for many years. Recently, it has gained a lot of interest with the advent of Web 2.0 and the enormous increase in the use of social networking applications, customer review sites, blogs, wikis, etc. Such media present features unique to the Web, in terms of shared authorship, multitude of userprovided tags, inherent connectivity between users and their posted items, and high update rate. All these characteristics could be exploited in order to mine interesting information about the dynamics of user's interactions. Recommender Systems allow people to find the resources they need by making use of the experience and opinions of their nearest neighbours. It has been shown that incorporating social network relationships (e.g., friendship) and respective opinions/ratings improves the prediction, and consequently the recommendation process [3]. Contrary to the initial works on user recommender systems for social networks that do not incorporate trust [5],[8], and following the paradigm of more recent research works [4],[5],[6],[7], our paper capitalizes on trust (and distrust) between people in order to assist members of a community to make decisions about other members of the same community.

In a nutshell, our contribution is a system for providing personalized user recommendations. The proposed system exploit positive and negative, time-dependent trust-related information, expressed either explicitly or implicitly.[1] The proposed system uses a collaborative reputation mechanism that captures and quantifies the users' connections and capitalize on trust propagation and on the dynamics of the social network. Using this mechanism, the system proposes new trust/distrust connections to the network's members. We should point out that the system can be applied to any type of social network, even in the absence of explicit trust connections, since in such cases only the implicit expressions of trust will be considered for the ranking and recommendation of users. Also, before giving the recommendations to the user, we are adding one more concept of profile matching [2] of users under consideration. This will help the user to increase the number of best matching and trustworthy connections.

\section{LITERATURE SURVEY}

The analysis of content and links in social networks has gained a lot of momentum, resulting in an increase of research in the related fields [1]. The largest body of work involving positive trust and/or trust propagation in the context of recommender systems has focused on item recommendations [9]. Time dynamics have been introduced by Walter et al. The notion of trust propagation through transitivity is employed, and, similarly to our paper, discounting takes place by multiplying trust values along paths.

Making new connections according to personal preferences is a crucial service in mobile social networking, where an initiating user can find matching users within physical proximity of him/her. According to the work in [2], FindU, a set of privacy-preserving profile matching schemes for proximity-based mobile social networks, is proposed. In FindU, an initiating user can find from a group of users the one whose profile best matches with his/her; to limit the risk of privacy exposure, only necessary and minimal information about the private attributes of the participating users is exchanged.

Online social networks experienced enormous growth in a small amount of time and users do spend respectable amount of their everyday life interacting about various topics with other peers. The case of interaction is expressed as direct messages or through application mechanisms that allow their authenticated users to communicate through these 
applications. The task of personalized recommendation requires the ability to predict which items will be considered interesting by the user. Such prediction is typically based on (1) content, i.e., recommending items with content that is "similar "to the content of the items already consumed by the users; (2) social networks, i.e., providing items related to people who are related to the user, either by explicit familiarity connection, or by some kind of similarity (e.g., by using similar tags, consuming similar documents, or having similar tastes as expressed in item rating). We note that we refer to social networks in their broad definition, i.e., networks of people; where connecting edges may represent any type of relationship, not only direct familiarity. Contentbased recommendation relies on the assumption that user interests are reflected by previous items they have consumed. This assumption has several drawbacks, among them the changes in user interests over time and the typical restriction to items similar to those already consumed.

A more generic model, which can be readily applied to any social medium, has been presented in the previous work [7]. Negative trust, previously introduced in different contexts, such as peer-to-peer networks, web recommender systems, and community discovery, has recently been introduced in the context of user recommendations in social networks [4], [11]. In this model, the trust of a user to another user is based on a personalized reputation rating, which quantifies explicit connections among users and implicit connections inferred from the interactions among users. Social networking sites allow users to articulate their social networks by adding other users to their "friend lists "[6].

Leskovecet al.[5] who try to predict positive and negative links in social networks using a machine-learning framework and ideas drawn from sociology have derived opposite results. Recommendations are based on aggregated social network information from various sources across the organization [8]. A more generic model, which can be readily applied to any social medium, has been presented in our previous work [7]. We defined both local and global metrics for user recommendations in social media that could be applied to any social media. However, in that work, we did not incorporate the notion of negative trust among users.

It has been shown that incorporating social network relationships (e.g., friendship) and respective opinions/ratings improves the prediction, and consequently the recommendation process [3], [6]. A similar line of work focuses on content ranking, which is consequently employed to recommend the top-ranked items (reviews, blogs, comments, tweets, etc.) to users. This is particularly important since the rapid increase in terms of content and users of social media shifts the problem of information search to that of information discovery.

Trust is often defined as the belief of an entity in the benevolence of another entity to act honestly and reliably in opposition to distrust [10].

\section{Table 1. Previous Related Work}

\begin{tabular}{|c|c|}
\hline Related Work & Objective \\
\hline $\begin{array}{l}\text { Ming Li, Shucheng Yu, } \\
\text { Ning Cao and Wenjing } \\
\text { Lou [2] }\end{array}$ & $\begin{array}{l}\text { This paper, proposes FindU, a set } \\
\text { of privacy-preserving profile } \\
\text { matching schemes for proximity- } \\
\text { based mobile social networks. In } \\
\text { FindU, an initiating user can find } \\
\text { from a group of users the one } \\
\text { whose profile best matches with } \\
\text { his/her; to limit the risk of } \\
\text { privacy exposure, only necessary } \\
\text { and minimal information about } \\
\text { the private attributes of the } \\
\text { participating users is exchanged. }\end{array}$ \\
\hline $\begin{array}{l}\text { I. Guy, N. Zwerdling, D. } \\
\text { Carmel, I. Ronen, E. } \\
\text { Uziel, S. Yogev, and S. } \\
\text { Ofek-Koifman [3] }\end{array}$ & $\begin{array}{l}\text { Study of personalized } \\
\text { recommendation of social } \\
\text { software items, including } \\
\text { bookmarked web-pages, blog } \\
\text { entries, and communities. The } \\
\text { focus is on recommendations that } \\
\text { are derived from the user's social } \\
\text { network. Social network } \\
\text { information is collected and } \\
\text { aggregated across different data } \\
\text { sources within our organization. } \\
\text { User recommendation was not } \\
\text { taken into consideration. }\end{array}$ \\
\hline $\begin{array}{l}\text { J. Chen, W. Geyer, C. } \\
\text { Dugan, M. J. Muller, and } \\
\text { I. Guy [5] }\end{array}$ & $\begin{array}{l}\text { Focuses on content ranking, } \\
\text { which is consequently employed } \\
\text { to recommend the top-ranked } \\
\text { items (reviews, blogs, comments, } \\
\text { tweets, etc.) to users. The main } \\
\text { objective was on item } \\
\text { recommendation. }\end{array}$ \\
\hline $\begin{array}{l}\text { I. Konstas, } \\
\text { Stathopoulos, and J. M. } \\
\text { Jose [6] }\end{array}$ & $\begin{array}{l}\text { People recommendations } \\
\text { designed to help users find } \\
\text { known, offline contacts and } \\
\text { discover new friends on social } \\
\text { networking sites. This considers } \\
\text { user recommendation, but the } \\
\text { trust between them was not } \\
\text { focused. }\end{array}$ \\
\hline $\begin{array}{l}\text { I. Guy, I. Ronen, and E. } \\
\text { Wilcox [8] }\end{array}$ & $\begin{array}{l}\text { This paper describes a novel UI } \\
\text { and system for providing users } \\
\text { with recommendations of people } \\
\text { to invite into their explicit } \\
\text { enterprise social network. The } \\
\text { recommendations are based on } \\
\text { aggregated information collected } \\
\text { from various sources across the } \\
\text { organization and are displayed in } \\
\text { a widget, which is part of a } \\
\text { popular enhanced employee } \\
\text { directory. }\end{array}$ \\
\hline J. Golbeck [9] & $\begin{array}{l}\text { In this work features of profile } \\
\text { similarity are investigated and } \\
\text { how those relate to the way users } \\
\text { determine trust is studied. } \\
\text { Through a controlled study, they } \\
\text { isolate several profile features } \\
\text { beyond overall similarity that } \\
\text { affect how much subjects trust a }\end{array}$ \\
\hline
\end{tabular}




\begin{tabular}{|l|l|}
\hline T. Grandison and M. & hypothetical users. \\
Sloman [10] & \begin{tabular}{l} 
This survey examines the various \\
definitions of trust in the \\
literature and provides a working \\
definition of trust for Internet \\
applications. The properties of \\
trust relationships are explained \\
and classes of different types of \\
trust identified in the literature \\
are discussed with examples. \\
$\begin{array}{l}\text { Some influential examples of } \\
\text { trust management systems are } \\
\text { described. }\end{array}$ \\
\hline J. Kunegis, A. \\
Bommatzsch, and C.
\end{tabular}$\quad \begin{array}{l}\text { This paper considered social } \\
\text { network analysis on graphs with } \\
\text { negative edge weights. They } \\
\text { studied the Slashdot Zoo, a social } \\
\text { network that is well-known for } \\
\text { containing negative links. The } \\
\text { study of the Slashdot Zoo showed } \\
\text { that the network exhibits } \\
\text { multiplicative transitivity, a } \\
\text { property of signed social } \\
\text { networks that can be summarized } \\
\text { by the phrase the enemy of my } \\
\text { enemy is my friend. They showed } \\
\text { that these methods for analysing a } \\
\text { network with negative edge } \\
\text { weights apply to large social } \\
\text { networks and reveal facts that } \\
\text { cannot be uncovered using } \\
\text { common, unsigned techniques. }\end{array}$ \\
\hline
\end{tabular}

\section{MOTIVATION}

Initial research on such recommendation systems in social networks like online social network, the blog sphere, social bookmarking applications do not incorporate the "trust" in the suggestions. Calculation of such kind of trust worthy recommendation is somewhat difficult because trust is also personalized in that it is subjective and affected by each user's personal beliefs, as well as those of members whom the user respects and trust, and this is the bottle neck problem. The main goal and challenge of the system is recommending personalized users to another user by matching their profiles as well as considering trust between them.

\section{PROPOSED SYSTEM}

The proposed recommender system is based on reputation mechanism that rates participants using observations, past experiences, and other user's view / opinion. In order to compute the reputation of each member, the several properties of trust such as, transitivity, personalization, and context are adopted and ideas from sociology axioms are drawn. Additionally, in order to address the social network dynamics, the element of time has been incorporated in the proposed system. To this direction, suggestion is given that reputation fades by time; thus, the positive (negative) reputation value of a user tends to zero unless new explicit or implicit trust (distrust) and liking (disliking) statements are added frequently. Finally, we assume that the context of trust is the same among community members. We exploit positive and negative, time-dependent trust-related information, expressed either explicitly or implicitly. We propose a collaborative reputation mechanism that captures and quantifies the user's connections and capitalizes on trust propagation and on the dynamics of the social network. Using this mechanism, the system proposes new trust/distrust connections to the network's members. We should point out that the system can be applied to any type of social network, even in the absence of explicit trust connections, since in such cases only the implicit expressions of trust will be considered for the ranking and recommendation of users. Robust reputation management is key feature of the proposed system based on trust awareness. Specifically, after processing information published on the network, connections (both explicit and implicit) that bear trust semantics between members are formed (phase 1), reputation ratings are estimated (phase 2), and personalized recommendations (both positive and negative) are generated (phase 3 ).

\subsection{Phase 1: (User connection formation)}

Our system differentiates between explicit trust/distrust bonds amongst users that carry strong trust semantics and implicit trust statements that form more transient user connections in the network. These user connection formation or trust bonds can be categorized as follows -

A] Explicit user to user connection: A user may explicitly relate to another user by forming trust or distrust connections. Such connections represent more permanent bonds between users (e.g., a friendship or collaboration in the real world).

B] Explicit user to item connection: In this type of connection, the user provides a like or dislike type of comment to a specific item published by another user. The semantics of opinion expression differ among applications.

C] Implicit user to item connection: In this each content item published by a user has a unique identifier and a timestamp, and may contain one or more hyperlinks that point to other content items inside the social network or items (URLs) on the web. Preference to an item is shown implicitly, for example, by sharing an article in Reddit or Facebook, by retweeting a post in Tweeter, by positively or negatively commenting on a user's post.

D] Implicit user to user connection: In this connection the user-to-item information is mapped to the user-to-user level and is aggregated in order to provide a single implicit user-touser connection. The main thing in our project is we consider the distrust connections though that are not supported in all social networks.

\subsection{Phase 2: (Reputation Rating Estimation)}

The proposed reputation rating mechanism captures the effect of time (e.g., freshness of links) by modelling the fact that more recent events [i.e., newly added explicit or implicit trust (distrust) and like (dislike) statements] should weigh more in the evaluation of the target user's overall reputation rating by the evaluator. The use of time information allows us to distinguish between users who attain a high reputation for a short time period and users who manage to maintain their reputation at a constantly high level. Thus, the social network's dynamic aspect is taken into account and is effectively addressed.

Following are the reputation rating systems A] Local Rating B] Collaborative Rating C] Transitivity of Trust D] Trust aware personalized recommendations: This is the last step in which personalized collaborative reputation ratings for all 
users who are connected directly / indirectly with the evaluator up to specific transitivity horizon considered.

\subsection{Contribution}

The proposed system considers the negative trust between users to help them getting connected to another trustworthy user. Before providing the list apart from these filters we can contribute one more step or we can say mode of filter i.e. profile matching. In this mode the proposed system can provide list of friends or enemies using set of privacy preserving profile matching schemes. In this the initiating user can find from group of users the one, whose profile best matches with his/her.

\subsection{Phase 3: (Recommendations Generations)}

Based on the overall reputation ratings of the social network members as assessed by the evaluator user, the proposed system generates personalized positive and/or negative user recommendations, which can be used to form new trust and/or distrust connections. Positive recommendations can be used from the members in order to connect to new people (in social networking sites), subscribe to new blogs (in the blogosphere), and share resources (in social bookmarking applications). On the other hand, in the case of negative recommendations, the model in essence generates a list of untrustworthy users. This personalized blacklist can be exploited by the recommender system in order to alert users when content items are published from such untrustworthy users and discourage them from linking or browsing such content, or filter it out from their content feed. Both types of recommendations could be exploited in order for a user to update his/her trust and distrust connections in the social network.

\section{CONCLUSION}

The previous work done mainly focused on the item and user recommendation without considering the trust relationship between them. Because of this, the security of user connection might be disturbed. Thus, we propose a trust aware user recommender system to make connections of social network trustworthy by giving positive and negative recommendations to the users while matching their profiles for strong connection. So that, positive recommendations will help in connecting trustworthy users while negative recommendations will alert users not to connect to the untrustworthy users and also making aware of the contents published by such user.

\section{REFERENCES}

[1] Magdalini Eirinaki, Malamati D. Louta,Member, IEEE, and Iraklis Varlamis,Member, IEEE. "A TrustAware System for Personalized User Recommendations in Social Networks" IEEE Trans. Systems, Man, And
Cybernetics: Systems, Vol. 44, No. 4, pp. 409 - 421 APRIL 2014

[2] Ming Li, Member, IEEE, Shucheng Yu, Member, IEEE, Ning Cao, Student Member, IEEE, and Wenjing Lou, Senior Member, IEEE. "Privacy-Preserving Distributed Profile Matching in Proximity-based Mobile Social Networks" IEEE Trans. Wireless Communications Vol.: 12 No.: 5 pp. 2024 - 2033 Year 2013

[3] I. Guy, N. Zwerdling, D. Carmel, I. Ronen, E. Uziel, S. Yogev, and S. Ofek-Koifman, "Personalized recommendation of social software items based on social relations," in Proc. 3rd ACM Conf. Recommender Syst., 2009, pp. 53-60.

[4] J. Leskovec, D. P. Huttenlocher, and J. M. Kleinberg, "Predicting positive and negative links in online social networks," inProc. 19th Int. Conf. World Wide Web, 2010, pp. 641-650.

[5] J. Chen, W. Geyer, C. Dugan, M. J. Muller, and I. Guy, "Make new friends, but keep the old: Recommending people on social networking sites," in Proc. SIGCHI Conf. Human Factors Comput. Syst., 2009, pp. 201-210.

[6] I. Konstas, V. Stathopoulos, and J. M. Jose, "On social networks and collaborative recommendation," in Proc. 32nd Int. ACM SIGIR Conf. Res. Develop. Inf. Retrieval, 2009, pp. 195-202.

[7] I. Varlamis, M. Eirinaki, and M. D. Louta, "Application of social network metrics to a trust-aware collaborative model for generating personalized user recommendations," in The Influence of Technology on Social Network Analysis and Mining(Lecture Notes in Social Networks Series), vol. 6, T. Ozyer, J. G. Rokne, G. Wagner, and A. H. P. Reuser, Eds. Berlin, Germany: Springer, 2013, pp. 49-74.

[8] I. Guy, I. Ronen, and E. Wilcox, "Do you know? : Recommending people to invite into your social network," in Proc. 14th Int. Conf. Intell. User Interfaces, 2009, pp. 77-86.

[9] J. Golbeck, "Trust and nuanced profile similarity in online social networks,"J. ACM Trans. Web, vol. 3, no. 4, pp. 1-33, 2009.

[10] T. Grandison and M. Sloman, "A survey of trust in internet applications," IEEE Commun. Surveys Tuts., vol. 3, no. 4, pp. 2-16, Oct. 2000.

[11] J. Kunegis, A. Lommatzsch, and C. Bauckhage, "The slashdot zoo: Mining a social network with negative edges," inProc. 18th Int. Conf. World Wide Web, 2009, pp. 741-750. 